\title{
Network pharmacology-based study to explore the mechanism of the Yiqi Gubiao pill in lung cancer treatment
}

\author{
ZHENG LI ${ }^{1,2}$, DAN XU ${ }^{1,2}$, JING JING ${ }^{1,2}$ and FENGSEN LI ${ }^{1,2}$ \\ ${ }^{1}$ Respiratory Department, The Traditional Chinese Medicine Hospital Affiliated to Xinjiang Medical University; \\ ${ }^{2}$ National Clinical Research Base of Traditional Chinese Medicine in Xinjiang, Urumqi, Xinjiang 830000, P.R. China
}

Received July 31, 2020; Accepted December 18, 2020

DOI: 10.3892/ol.2021.12583

\begin{abstract}
Lung cancer (LC) has been one of the most prevalent and fatal malignancies in the past 5 years. Yiqi Gubiao pills have a good clinical effect against LC. However, their complex composition limits proper understanding of their pharmacological mechanism. Therefore, the present study aimed to systemically explore the underlying mechanisms of Yiqi Gubiao pills in treatment of LC. The network pharmacology approach was employed to identify the active ingredients and LC targets associated with Yiqi Gubiao pills. Prediction of potential active ingredients and action targets was then conducted through protein-protein interaction (PPI), Gene Ontology and Kyoto Encyclopedia of Genes and Genomes pathway analyses. In vitro experiments were then performed to further verify the mechanism of action of Yiqi Gubiao pills, revealing that the anti-LC effects were mediated by regulating the expression of IL6, TP53, albumin (ALB), MAPK3 and AKT1. In total, 102 active ingredients and 229 targets of Yiqi Gubiao pills were identified. The PPI network further revealed that AKT1, TP53, ALB, IL6 and MAPK3 were the top five hub genes associated with LC treatment. Targets of the Yiqi Gubiao pills were mainly enriched in the PI3K-Akt and Advanced glycation end products (AGE)-receptors for AGEs (RAGE) signaling pathways. Overall, network pharmacology deciphered the active ingredients and potential targets of the Yiqi Gubiao pills. Yiqi Gubiao pills partially inhibited the progression of $\mathrm{LC}$ by regulating the expression of hub genes (AKT1, TP53, ALB, IL6 and MAPK3) through the PI3K-Akt and AGE-RAGE signaling pathways. The findings of the present study may provide a theoretical basis for the clinical application of Yiqi Gubiao pills in LC treatment.
\end{abstract}

Correspondence to: Dr Fengsen Li, Respiratory Department, The Traditional Chinese Medicine Hospital Affiliated to Xinjiang Medical University, 116 Huanghe Road, Urumqi, Xinjiang 830000, P.R. China

E-mail: fengsen602@163.com

Key words: network pharmacology, Yiqi Gubiao pill, hub gene, verification experiment

\section{Introduction}

Incidences and deaths caused by lung cancer (LC) across the world have been on the rise (11.6 and 18.4\%) $(1,2)$. As such, the disease has become an urgent public health concern. In China, the incidence of LC in 2015 was $733 / 100,000$ individuals. Its mortality rate in the same year was $610 / 100,000$ individuals, thus causing the disease to rank first among causes of cancer-associated deaths. Patients with LC often face physical, emotional, and financial distress, which not only affects their mental health but may also impose financial and social burdens to families (3). Currently, chemotherapy is the common clinical treatment method used to treat LC. However, this method also damages normal cells, thus leading to other adverse reaction, such as nausea, vomiting, anorexia and diarrhea (4). As such, patients with LC undergoing chemotherapy have been using tradition Chinese medicine as an adjuvant therapy.

The Yiqi Gubiao pill is the most used drug in treatment of lung-related diseases, such as chronic obstructive pulmonary disease (COPD), in Xinjiang Uygur Autonomous Region Hospital (5). The pill is composed of 13 traditional Chinese medicines namely, Dangshen [DS, Codonoposis pilosula (Franch)], Baizhu (BZ, Atractylodes macrocephala), Fuling [FL, Poriacocos (Schw) Wolf], Chenpi (CP, Citrus reticulata Blanco), Banxia [BX, Pinelliaternata (Thunb) Breit], Yiyiren [YYR, Coixlacryma-jobi L.var.mayuen (Roman) Stapf], Fuxiaomai (FXM, Triticum aestivum L.), Zisu (ZS, Perilla frutescens (L.) Britt), Kuandonghua (KDH, Tussilago farfara L.), Huangqin (HQ, Scutellaria baicalensis Ceorgi), Yibeimu (YBM, Fritillariapallidiflora Schrenk), Pibaye [Eriobotrya japonica (Thunb) Lindl] and Fangfeng [FF, Saponshnikovia divaricate (Turcz) Schischk]. Previous studies have reported that Yiqi Gubiao pills function in COPD treatment via the JAK/STAT pathway to prolong the stable period of COPD as well as reduce the number of acute episodes $(5,6)$.

Yiqi Gubiao pills are multi-component traditional Chinese medicine (TCM) and thus have multi-target properties. As such, it is challenging to decipher its underlying mechanism(s) in treating LC. Network pharmacology integrates multi-disciplinary technologies, such as systems biology, multi-directional pharmacology, network analysis and computational biology, to help in investigate the mechanism of TCMs $(7,8)$. The relationships between drugs and diseases are revealed at the 
system level by constructing a multi-level structure network of 'Disease-Phenotype-Gene-Drug'.

Herein, network pharmacology was employed to analyze the complex network relationships among the multi-components and multi-target properties of Yiqi Gubiao and LC. Biological experiments were set up and used to identify and verify the key genes involved in Yiqi Gubiao adjuvant treatment of LC. This revealed the mechanism of action of Yiqi Gubiao pills, thus providing a theoretical basis for its clinical application (Fig. 1).

\section{Materials and methods}

Screening the active ingredients of the Yiqi Gubiao pill. A search for the ingredients of the Yiqi Gubiao pill was conducted using the Chinese Medicine System Pharmacology Analysis Platform (http://lsp.nwu.edu.cn/tcmsp.php) and the SymMap database (http://www.symmap.org) (9). An oral bioavailability $(\mathrm{OB}) \geq 30 \%$ and drug-likeness (DL) $\geq 0.18$ were set as the cut-off values for choosing the active ingredients.

Collection of targets. A search of the validated targets of the active ingredients of the Yiqi Gubiao pill was performed using the Herbal Ingredients' Targets Database (HIT, http://lifecenter.sgst.cn/hit/) (10). The PubChem database (https://pubchem.ncbi.nlm.nih.gov/) was used to query the SMILES number of the active compounds. The SMILES numbers were then imported into the Similarity Ensemble Approach database (SEA, http://sea.bkslab.org) (11) to predict the targets of the active ingredients. Targets obtained in the SEA and HIT databases were used for subsequent analysis. The Online Mendelian Inheritance in Man (OMIM, https://omim.org) (12), Pubmed-Gene (https://www.ncbi. nlm.nih.gov/pubmed/gene) (13) and Genecards (https://www. genecards/org) bioinformatics tools were employed to identify LC-related targets. All targets were then calibrated to correspond to the official gene name using the UniProt database (https://www.uniprot.org/) (14).

Protein-protein interaction (PPI) network construction and analysis. The online website, Funrich (http://funrich. org/index.html) was used to overlap the active ingredients and disease targets to obtain the common targets. These common targets were considered as the potential key targets of the Yiqi Gubiao pills in LC treatment. Gene targets of the Yiqi Gubiao pill and LC co-acting targets were then imported into the online STRING platform (https://string-db.org) to obtain their protein interaction data. The score was set at $>0.7$ to filter the information. In addition, the Cytoscape 3.7.2 platform (15) was used to build the protein-protein interaction (PPI) network, while the plug-in cytohubba was employed to identify the top five genes based on degree values (degree $>128$ ). These genes were regarded as the hub genes. Finally, the information of the target hub genes was identified using the DisGeNET database (version 5; http://www.disgenent.org/web/DisGeNet/menu).

Gene Ontology (GO) and Kyoto Encyclopedia of Genes and Genomes (KEGG) enrichment analysis. The GO tool has several functions such as molecular function (MF), biological process (BP) and cellular component (CC), which are applied to the biological mechanism of high-throughput genome or transcriptome data identification (16). On the other hand, the KEGG database is a functional and biological platform for identifying candidate genes. Herein, the 'clusterProfiler' package in the R software (version 3.5.1) (17) was used for the GO function and KEGG pathway analysis of targets in the PPI network.

Source of drugs and reagents. The Yiqi Gubiao pills were purchased from the Xinjiang Uygur Autonomous Region Hospital (Xinjiang, batch no. 20121212). They were decocted twice with $\mathrm{ddH}_{2} \mathrm{O}$ for $30 \mathrm{~min}$ and their filtrates mixed and condensed to $2 \mathrm{~g}$ crude $\mathrm{drug} / \mathrm{ml}$. The Cell Counting Kit- 8 (CCK-8) was purchased from Invitrogen (Thermo Fisher Scientific, Inc.) while AKT1, ALB, GAPDH, IL6 and MAPK3 antibodies were purchased from Cell Signaling Technology, Inc.

Cells lines and cell cultures. The human non-small cell (NSC) LC cell line A549 was used in the present study. It was purchased from the Cell Bank of Type Culture Collection of Chinese Academy of Sciences. The cells were cultured in Dulbecco's modified Eagle's medium (DMEM) supplemented with $10 \%$ fetal calf serum (FCS) and $100 \mathrm{U} / \mathrm{ml}$ penicillin-streptomycin (all Hyclone; Cyvita), and then maintained in a cell culture incubator set at $37^{\circ} \mathrm{C}$ and $5 \% \mathrm{CO}_{2}$.

Cell viability assay. The A549 cells were seeded into a 96 -well plate at a density of $5 \times 10^{3}$ cells/well and incubated in a cell culture incubator set at $37^{\circ} \mathrm{C}, 5 \% \mathrm{CO}_{2}$ and saturated humidity for $24-\mathrm{h}$ prior to treatment. Cells were then randomly divided into five groups: Control group and Yiqi Gubiao pill groups $(50,25,12.5$ and $6.25 \mu \mathrm{g} / \mathrm{ml})$. The supernatant of the medium was aspirated and discarded, followed by addition of $100 \mu 1$ 10\% CCK-8 phenol red-free DMEM medium added to the cells. The cultures were left to stand for $2 \mathrm{~h}$ and their absorbance measured at a wavelength of $450 \mathrm{~nm}$ using as a microplate reader (BioTek Instruments). Cell viability (\% of control) was calculated using the formula: (Absorbance of drug treatment group/absorbance of control) x100\%.

Reverse-transcription quantitative $(R T-q) P C R$. Total RNA was isolated from the A549 cells treated with varying concentrations of Yiqi Gubiao pills (50, 25, 12.5 and $6.25 \mu \mathrm{g} / \mathrm{ml}$ ) using TRIzol ${ }^{\circledR}$ reagent (Invitrogen; Thermo Fisher Scientific, Inc.). The RNA was then reverse transcribed to cDNA and used as a template in the RT-qPCR reaction to amplify the hub genes using the PrimeScript RT Reagent kit with gDNA Eraser (cat no. RR037A; Takara Biotechnology Co., Ltd.), according to the manufacturer's. qPCR was subsequently performed using the SYBR Premix Taq $^{\text {TM }}$ kit (cat. no. DRR820A; Takara Biotechnology Co., Ltd.), on a Agilent Mx3005P (Agilent Technologies, Inc.), according to the manufacturer's instructions. The following thermocycling conditions were used: 1 cycle at $95^{\circ} \mathrm{C}$ for $10 \mathrm{~min}$, and 40 cycles at $95^{\circ} \mathrm{C}$ for $30 \mathrm{sec}, 95^{\circ} \mathrm{C}$ for $5 \mathrm{sec}$ and $60^{\circ} \mathrm{C}$ for $30 \mathrm{sec}$. The primer sequences used to amplify the hub genes are listed in Table I. GADPH was used as an internal control. Reactions were conducted in triplicate for each sample and the mean value determined for subsequent 

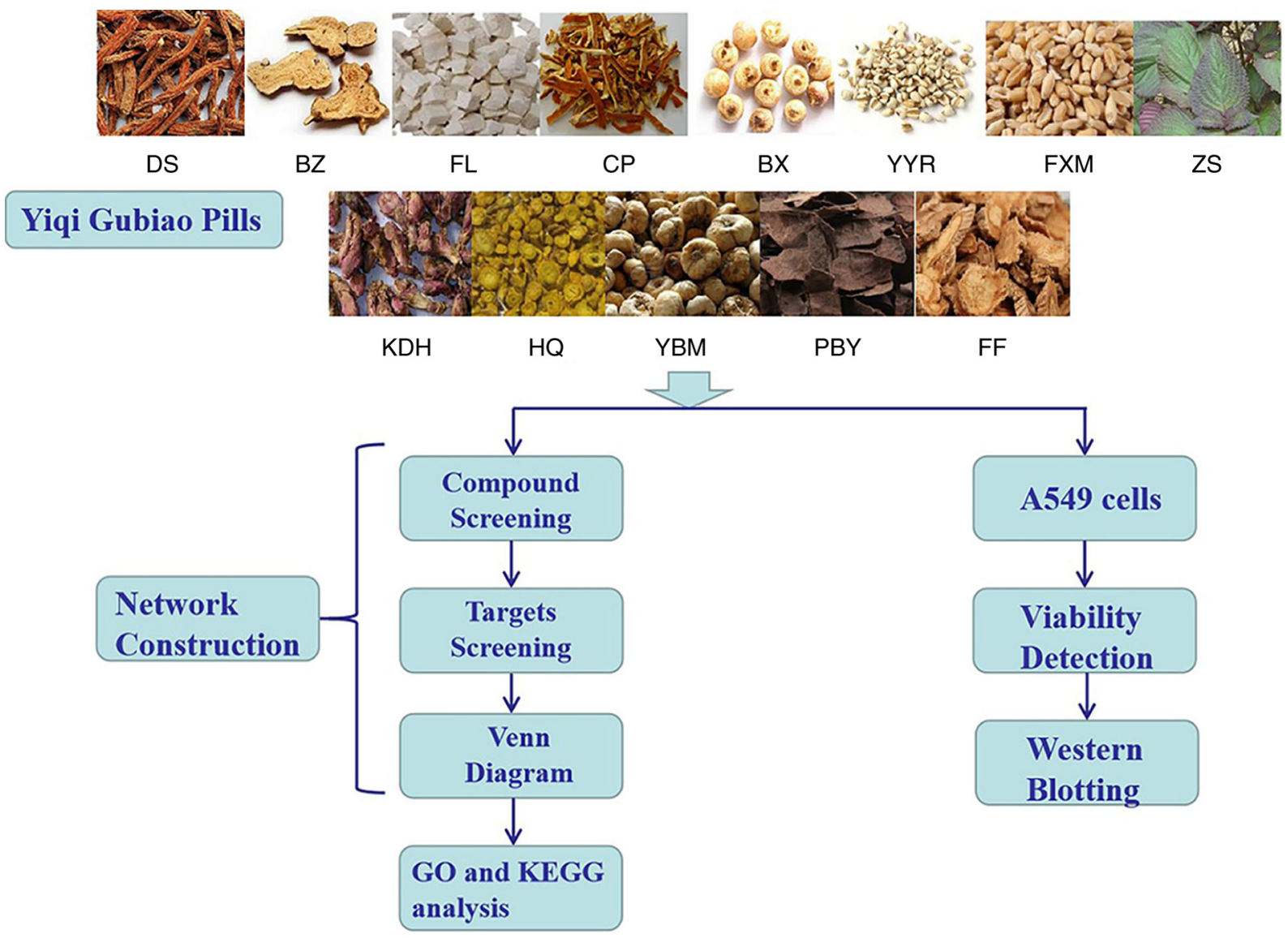

Figure 1. Complete flow diagram of the study. DS, Danshen; BZ, Baizhu; FL, Fuling; CP, Chenpi; BX, Banxia; YYR, Yiyiren; FXM, Fuxiaomai; ZS, Zisu; KDH, Kuandonghua; HQ, Huangqin; YBM, Yibeimu; PBY, Pibaye; FF, Fangfeng.

Table I. Primer sequences used for the quantitative PCR.

\begin{tabular}{ll}
\hline Name & \multicolumn{1}{c}{ Sequence, 5' $\rightarrow 3^{\prime}$} \\
\hline AKT1 & F: CCTCCACGACATCGCACTG \\
& R: TCACAAAGAGCCCTCCATTATCA \\
ALB & F: GGGGTGTGTTTCGTCGAGAT \\
& R: AGGCAATCAACACCAAGGCT \\
IL-6 & F: GAACTCCTTCTCCACAAGCGCCTT \\
& R: CAAAAGACCAGTGATGATTTCACCAGG \\
MAPK3 & F: TCCGCCATGAGAATGTTATAGGC \\
& R: GGTGGTGTTGATAAGCAGATTGG \\
TP53 & F: GGAGCCGCAGTCAGATCCTAG \\
& R: CAAGGGGGACAGAACGTTG \\
GAPDH & F: GAAGGTCGGAGTCAACGGATTT \\
& R: CCTGGAAGATGGTGATGGGATT
\end{tabular}

F, forward; R, reverse.

analysis. The relative expression of mRNA was calculated using the $2^{-\Delta \Delta \mathrm{Cq}}$ method (18).

Western blot analysis. A549 cells were seeded in 6-well plates at a density of $5 \times 10^{3}$ cells/well for $24-\mathrm{h}$ before treatment. The cells were then treated with varying concentrations of Yiqi Gubiao $(50,25,12.5$ and $6.25 \mu \mathrm{g} / \mathrm{ml})$. They were then lysed in RIPA buffer (Thermo Fisher Scientific, Inc.) containing $50 \mathrm{mM}$ Tris- $\mathrm{HCl}$ (pH 7.5), $150 \mathrm{mM} \mathrm{NaCl}, 1 \mathrm{mM}$ EDTA, $1 \mathrm{mM}$ $\mathrm{MgCl} 2,0.5 \%$ Triton $\mathrm{X}-100$, phosphatase inhibitor mix (1 mM $\mathrm{NaF}, 1 \mathrm{mM}$ Na3VO4, and $1 \mathrm{mM} \beta$-glycerol phosphate) and centrifuged at 5,600 $\mathrm{x}$ g for $5 \mathrm{~min}$ at $4^{\circ} \mathrm{C}$, which contained the proteins. Protein quantification was measured using the BCA kit (cat. no. p0006; Beyotime Biotechnology, Inc.), and a microplate reader. The protein lysates $(50 \mu \mathrm{g})$ were mixed with 3x sample buffer solution and heated for $5 \mathrm{~min}$. Protein samples were loaded on a $12 \%$ SDS-gel and resolved using SDS-PAGE for $3 \mathrm{~h}$. Resolved proteins were transferred onto nitrocellulose membranes and blocked with $0.01 \%$ Tween-20 containing 5\% skimmed milk powder for $4 \mathrm{~h}$ at room temperature. The membranes were incubated with primary antibodies against AKT1 (cat. no. AF4718), ALB (cat no. DF6396), TP53 (cat. no. DF7238), IL6 (cat. no. DF6087), MAPK3 (cat. no. BF0528) and GADPH (cat. no. AF0911) (all for 1:1,000 and purchased from Affinity Co., Ltd.), for $24 \mathrm{~h}$ at $4^{\circ} \mathrm{C}$. The membranes were washed with TBST buffer $(10 \mathrm{mM}$ Tris- $\mathrm{HCl}$ (pH 7.5), $150 \mathrm{mM} \mathrm{NaCl}, 0.1 \%$ Tween-20) and subsequently incubated with anti-rabbit IgG secondary antibodies with TBST solution (1:3,000; cat. no. S0001; Affinity Co., Ltd.) for $1 \mathrm{~h}$ at room temperature. The membranes were re-washed with TBST and the protein bands were visualized using enhanced chemiluminescence reagent (SuperSignal Western Pico 
Table II. Active ingredients of Yiqi Gubiao pills.

\begin{tabular}{|c|c|c|c|c|}
\hline Molecule name & CAS & OB & DL & Herb \\
\hline Cavidine & $32728-75-9$ & 35.64 & 0.81 & $\mathrm{BX}$ \\
\hline Coniferin & $109664-02-0$ & 31.11 & 0.32 & $\mathrm{BX}$ \\
\hline Cycloartenol & 469-38-5 & 38.69 & 0.78 & $\mathrm{BX}$ \\
\hline Gondoic acid & $5561-99-9$ & 30.70 & 0.20 & $\mathrm{BX}$ \\
\hline Stigmasterol & $83-48-7$ & 43.83 & 0.76 & BX, DS, HQ, YYR \\
\hline Baicalein & $491-67-8$ & 33.52 & 0.21 & BX, HQ \\
\hline Baicalin & $31564-28-0$ & 40.12 & 0.75 & BX, HQ \\
\hline Didehydrotuberostemonine & $106861-40-9$ & 51.90 & 0.74 & BZ \\
\hline Formononetin & $485-72-3$ & 69.67 & 0.21 & $\mathrm{BZ}$ \\
\hline Malkangunin & $52691-06-2$ & 57.71 & 0.63 & $\mathrm{BZ}$ \\
\hline Stemotinine & $85644-15-1$ & 38.69 & 0.46 & $\mathrm{BZ}$ \\
\hline Suchilactone & $50816-74-5$ & 57.51 & 0.56 & $\mathrm{BZ}$ \\
\hline$\alpha$-Amyrin & $638-95-9$ & 39.51 & 0.76 & $\mathrm{BZ}$ \\
\hline Naringenin & $153-18-4$ & 59.29 & 0.21 & $\mathrm{CP}$ \\
\hline Nobiletin & $10236-47-2$ & 61.67 & 0.78 & $\mathrm{CP}$ \\
\hline Pectolinarin & 28978-02-1 & 47.62 & 0.65 & $\mathrm{CP}$ \\
\hline Aurantiamide acetate & $56121-42-7$ & 58.38 & 0.52 & DS \\
\hline Daturilin & $111950-78-8$ & 50.37 & 0.77 & DS \\
\hline Frutinone A & $38210-27-4$ & 65.9 & 0.34 & $\mathrm{DS}$ \\
\hline Glycitein & $40957-83-3$ & 50.48 & 0.24 & DS \\
\hline Luteolin & 491-70-3 & 36.16 & 0.25 & $\mathrm{DS}, \mathrm{ZS}$ \\
\hline Perlolyrine & 29700-20-7 & 65.95 & 0.27 & DS \\
\hline Sitosterol & $83-46-5$ & 36.91 & 0.75 & DS \\
\hline Spinasterol & $481-18-5$ & 42.98 & 0.76 & DS \\
\hline Spinoside A & $524-40-3$ & 39.97 & 0.4 & $\mathrm{DS}$ \\
\hline Taraxasteryl palmitate & $29803-90-5$ & 33.84 & 0.31 & DS \\
\hline Taraxerol & $22076-46-6$ & 38.40 & 0.77 & $\mathrm{DS}$ \\
\hline Ammidin & 482-44-0 & 34.55 & 0.22 & $\mathrm{FF}$ \\
\hline Anomalin & 81740-07-0 & 59.65 & 0.66 & FF \\
\hline Decursin & $5928-25-6$ & 39.27 & 0.38 & $\mathrm{FF}$ \\
\hline Mandenol & $544-35-4$ & 42.00 & 0.19 & FF,YYR \\
\hline Marmesin & $13849-08-6$ & 50.28 & 0.18 & $\mathrm{FF}$ \\
\hline Nodakenetin & 495-32-9 & 68.62 & 0.18 & FF \\
\hline Phellopterin & $2543-94-4$ & 40.19 & 0.28 & FF \\
\hline Prangenidin & $642-05-7$ & 36.31 & 0.22 & $\mathrm{FF}$ \\
\hline Wogonin & $632-85-9$ & 30.68 & 0.23 & FF, HQ \\
\hline$\beta$ Sitosterol/sitosterol & $83-46-5$ & 36.91 & 0.75 & $\begin{array}{c}\text { FF, BZ, BX, CP, FXM, } \\
\text { HQ, KDH, YYR, ZS, PBY }\end{array}$ \\
\hline Ellipticine & $519-23-3$ & 30.82 & 0.28 & $\mathrm{FL}$ \\
\hline Hederagenin & $465-99-6$ & 36.91 & 0.75 & FL \\
\hline Pachymic acid & 29070-92-6 & 33.63 & 0.81 & FL \\
\hline Poricoic acid A & $137551-38-3$ & 30.61 & 0.76 & FL \\
\hline Poricoic acid B & $137551-39-4$ & 30.52 & 0.75 & FL \\
\hline Poricoic acid C & $151200-89-4$ & 38.15 & 0.75 & FL \\
\hline Trametenolic acid & $24160-36-9$ & 38.71 & 0.80 & $\mathrm{FL}$ \\
\hline Acacetin & 480-44-4 & 34.97 & 0.24 & HQ \\
\hline Carthamidin & $479-54-9$ & 41.15 & 0.24 & HQ \\
\hline Coptisine & $3486-66-6$ & 30.67 & 0.86 & HQ \\
\hline Dihydrooroxylin & 18956-18-8 & 66.06 & 0.23 & HQ \\
\hline Diop & $25103-50-8$ & 43.59 & 0.39 & HQ \\
\hline Epiberberine & 1816598 & 43.09 & 0.78 & HQ \\
\hline Glucobrassicin & $4356-52-9$ & 53.64 & 0.48 & HQ \\
\hline
\end{tabular}


Table II. Continued.

\begin{tabular}{|c|c|c|c|c|}
\hline Molecule name & CAS & $\mathrm{OB}$ & $\mathrm{DL}$ & Herb \\
\hline Glyceryl linolenate & $18465-99-1$ & 38.13 & 0.31 & HQ \\
\hline Moslosooflavone & $3570-62-5$ & 44.09 & 0.25 & HQ \\
\hline Neobaicalein & $55084-08-7$ & 104.34 & 0.44 & HQ \\
\hline Norwogonin & $4443-09-8$ & 39.40 & 0.21 & HQ \\
\hline Panicolin & $41060-16-6$ & 76.26 & 0.29 & HQ \\
\hline Rivularin & $70028-59-0$ & 37.94 & 0.37 & HQ \\
\hline Salvigenin & $19103-54-9$ & 49.07 & 0.33 & HQ \\
\hline Diosgenin & 512-04-9 & 80.87 & 0.81 & $\mathrm{KDH}$ \\
\hline Femara & $112809-51-5$ & 66.09 & 0.20 & $\mathrm{KDH}$ \\
\hline Kaempferol & $520-18-3$ & 41.88 & 0.24 & KDH, PBY \\
\hline Quercetin & $117-39-5$ & 46.43 & 0.28 & KDH, PBY, BX \\
\hline Senkirkine & $2318-18-5$ & 56.16 & 0.41 & $\mathrm{KDH}$ \\
\hline Tussilagin & $104012-37-5$ & 61.12 & 0.38 & $\mathrm{KDH}$ \\
\hline Cinchonain 1a & $85081-24-9$ & 30.12 & 0.93 & PBY \\
\hline Ellagic acid & 476-66-4 & 43.06 & 0.43 & PBY \\
\hline Eugenol & $97-53-0$ & 56.24 & 0.32 & PBY \\
\hline Isorhamnetin & $480-19-3$ & 49.60 & 0.31 & PBY \\
\hline Mairin & $472-15-1$ & 55.38 & 0.78 & PBY \\
\hline Cyclopamine & $4449-51-8$ & 55.42 & 0.82 & YBM \\
\hline Peimisine & 19773-24-1 & 57.40 & 0.81 & YBM \\
\hline Pelargonidin & 134-04-3 & 37.99 & 0.21 & YBM \\
\hline Siraitic acid A & $183374-15-4$ & 41.52 & 0.85 & YBM \\
\hline Verticinone & 1357-77-3 & 60.07 & 0.67 & YBM \\
\hline Yibeinoside C & $157536-48-6$ & 37.72 & 0.83 & YBM \\
\hline Sitosterol $\alpha 1$ & 474-40-8 & 43.28 & 0.78 & YYR \\
\hline (+)-Catechin & $154-23-4$ & 54.83 & 0.24 & $\mathrm{ZS}$ \\
\hline Beta-carotene & $7235-40-7$ & 37.18 & 0.58 & $\mathrm{ZS}$ \\
\hline Cyanin & $523-42-2$ & 47.42 & 0.76 & $\mathrm{ZS}$ \\
\hline
\end{tabular}

OB, oral bioavailability; DL, drug-likeness; DS, Danshen; BZ, Baizhu; FL, Fuling; CP, Chenpi; BX, Banxia; YYR, Yiyiren; FXM, Fuxiaomai; ZS, Zisu; KDH, Kuandonghua; HQ, Huangqin; YBM, Yibeimu; PBY, Pibaye; FF, Fangfeng.

Chemiluminescent Substrate; Pierce; Thermo Fisher Scientific, Inc.) and quantified using the FluorChem FC2 Imaging System (ProteinSimple). Densitomery analysis was performed using Image-Pro Plus 6.0 software (National Institutes of Health).

Statistical analysis. GraphPad Prism 8.0 software (GraphPad Software, Inc.) was used for statistical analysis and graphing of the data. All experiments were performed in triplicate and data are presented as the mean \pm standard error of the mean. Unpaired Student's t-tests were used to compare the difference between two groups, while one-way ANOVA was used to compare differences among $\geq 3$ groups, followed by Tukey's post hoc test. $\mathrm{P}<0.05$ was considered to indicate a statistically significant difference.

\section{Results}

Collection of active ingredients in Yiqi Gubiao pills. Yiqi Gubiao pills had 102 active ingredients, including 7 components of BZ, 8 components of BX, 4 components of $\mathrm{CP}$,
12 components of DS, 11 components of FF, 7 components of FL, 2 compounds of FXM, 19 components of HQ, 8 components of KDH, 8 components of PBY, 6 compounds of YBM, 4 components of YYR and 4 components of ZS (Table II).

Collection of targets. The targets of the active ingredients were predicted based on the principle of structural similarity. They included 174 targets of BZ, 311 targets of BX, 140 targets of BX, 246 targets of DS, 122 targets of FF, 159 targets of FL, 37 targets of FXM, 203 targets of HQ, 243 targets of KQH, 261 targets of PPY, 54 targets of YBM, 109 targets of YYR and 173 targets of ZS components (Data SI). Further to this, 7,446 targets associated with LC were detected in the OMIM database, 22,400 targets were detected in the Pubmed-Gene Database, and 3,007 targets in the Genecards Database. Intersection of the targets obtained from the three databases results in 2,413 targets.

PPI network construction and analysis. The predicted targets of Yiqi Gubiao pills were compared with those 


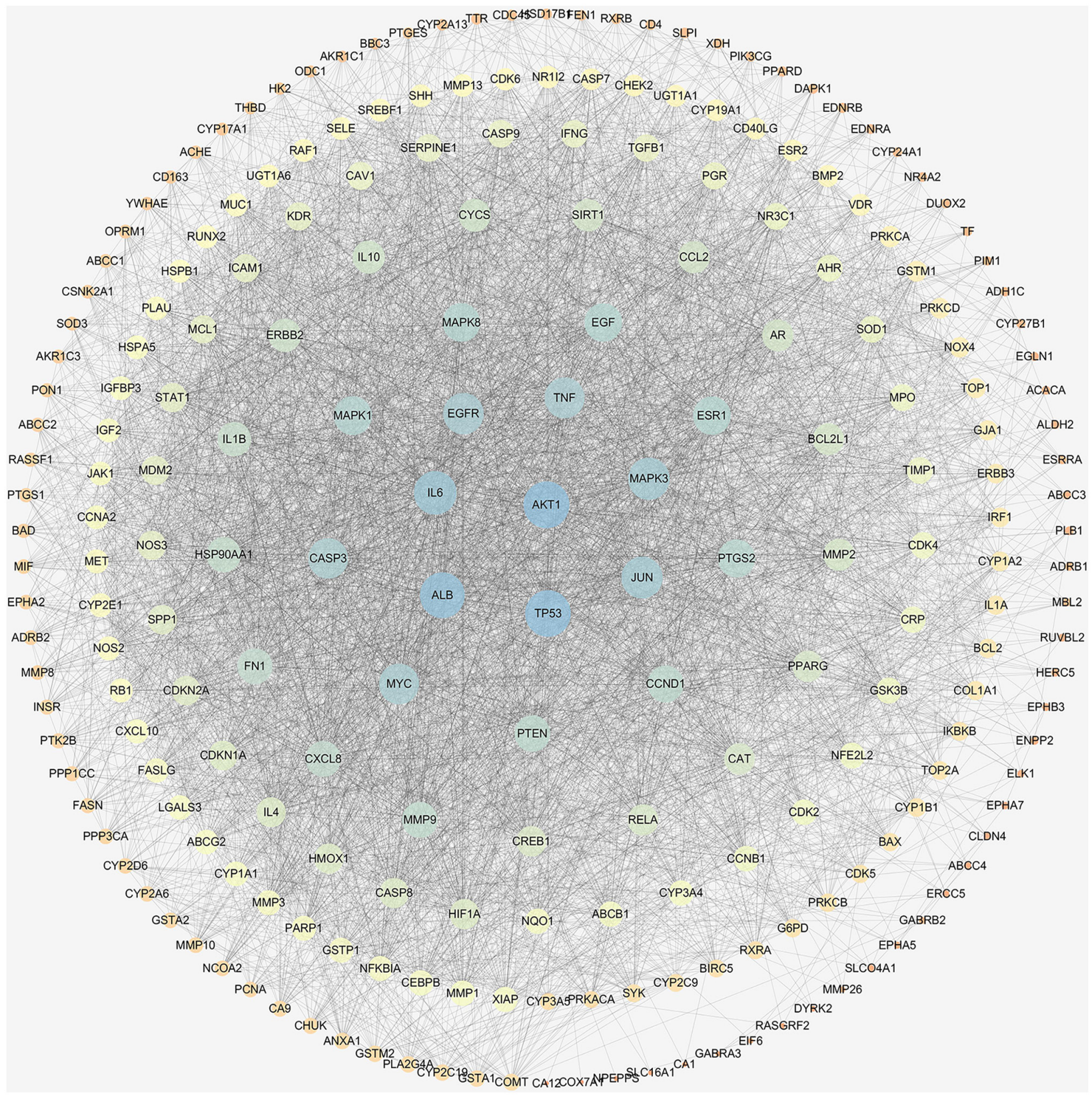

Figure 2. Protein-protein interaction analysis of potential targets genes.

Table III. Protein classes of key targets.

\begin{tabular}{llllr}
\hline Gene name & UniProt ID & \multicolumn{1}{c}{ Target } & \multicolumn{1}{c}{ Protein class } & Degree \\
\hline AKT1 & P31749 & AKT serine/threonine kinase 1 & Calcium-binding protein; kinase; & 149 \\
& & & transfer/carrier protein transderase & 148 \\
TP53 & P04637 & Tumor protein p53 & Transcription factor & 128 \\
MAPK3 & P27361 & Mitogen-activated protein kinase 3 & Kinase; transferase & 134 \\
IL6 & P05231 & Interleukin 6 & None & 144 \\
ALB & P02768 & Albumin & Transporter & \\
\hline
\end{tabular}

related to LC. Finally, 229 anti-LC potential targets of the Yiqi Gubiao pills were selected. These included AKT1, TP53, IL-6, ALB and MAPK3, among others. A PPI network composed of the 229 targets contained 227 nodes and 4,810 interaction edges (Fig. 2). The top five hub genes where protein kinase B (AKT1) (degree=149), TP53 (degree $=148)$, ALB (degree=144), IL6 (degree $=134)$, and MAPK3 (degree=128) (Table III). 
A

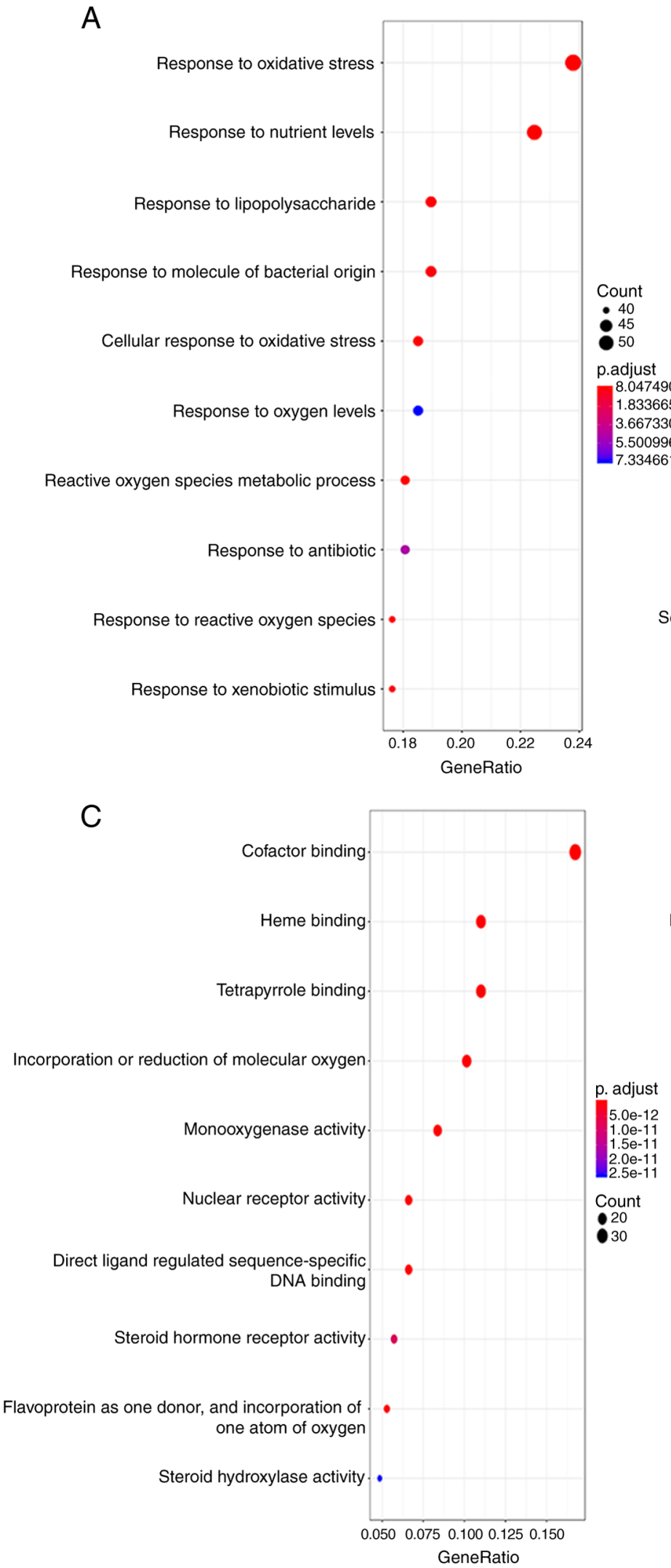

B
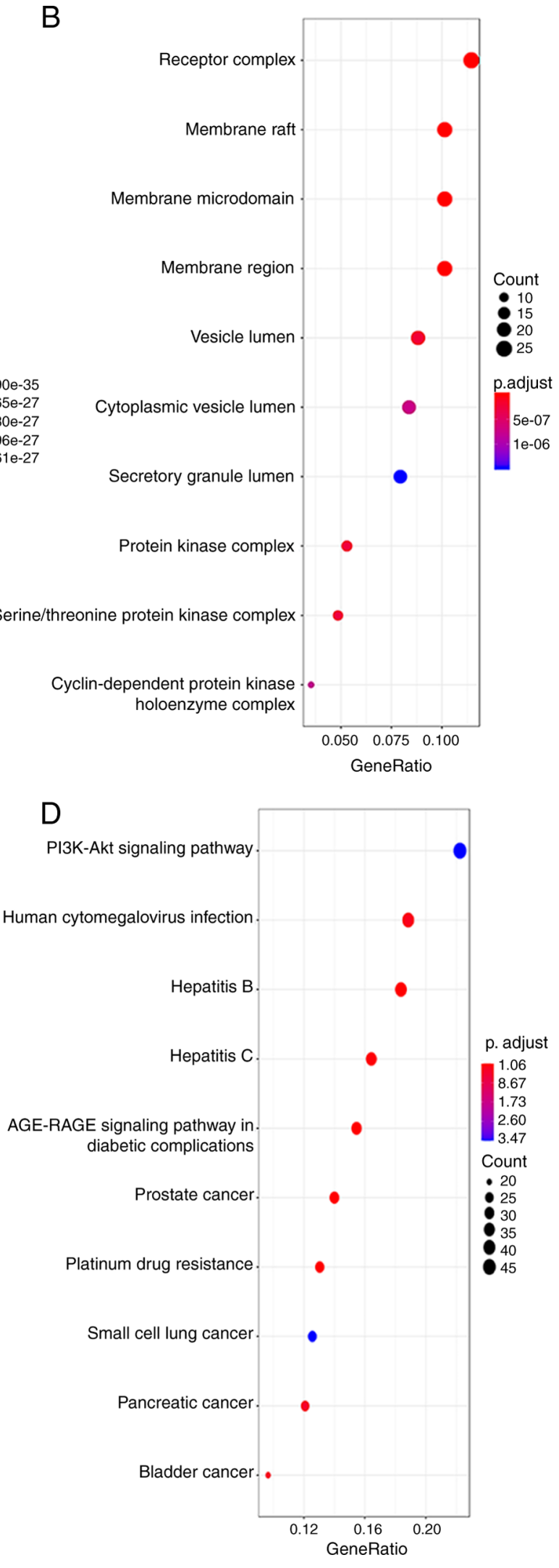

Figure 3. Gene Ontology and KEGG analysis of potential targets genes. (A) Biological process. (B) Cellular component. (C) Molecular function. (D) KEGG. KEGG, Kyoto Encyclopedia of Genes and Genomes.

GO and KEGG enrichment analysis. GO enrichment and KEGG pathway analysis was performed using the 227 genes in the PPI network. An adjusted $\mathrm{P}<0.05$ was set as the screening value. In $\mathrm{CC}$ enrichment analysis, the genes were mainly enriched in the signaling pathways such as 'membrane raft' and 'membrane microdomain' signaling pathways (Fig. 3B). 


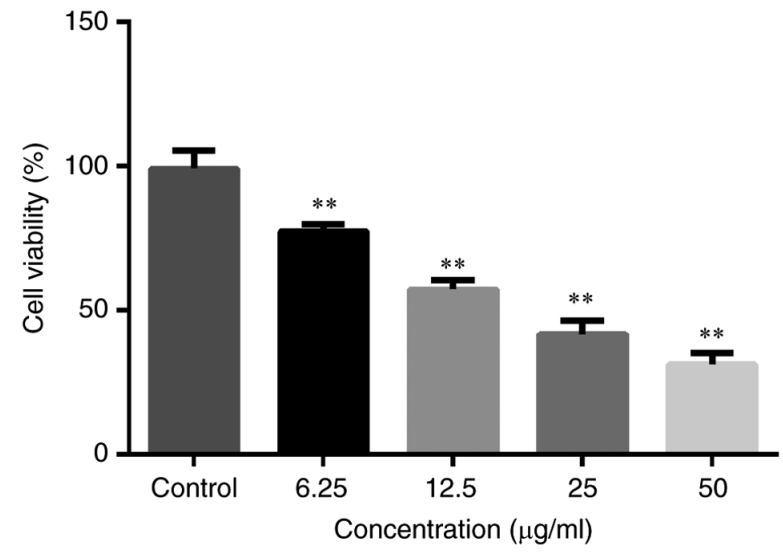

Figure 4. Yiqi Gubiao pills inhibit A549 cell proliferation. $n=3 .{ }^{* *} \mathrm{P}<0.01$ vs. control.

In BP, the genes were involved in response 'oxidative stresses', 'nutrient levels' and 'lipopolysaccharides'. In MF, the genes were mainly involved in binding activities, such as 'heme binding' (Fig. 3C). KEGG pathway analysis further revealed that the 'PI3K-Akt' and 'AGE-RAGE' signaling pathways were the most common pathways related to LC treatment using Yiqi Gubiao pills (Fig. 3D).

Cell viability. Viability of cells in the Yiqi Gubiao pill groups $(50,25,12.5$ and $6.25 \mu \mathrm{g} / \mathrm{ml})$ decreased significantly in a concentration-dependent manner compared with those of the control group (all $\mathrm{P}<0.01$; Fig. 4).

mRNA suppression and protein expression of the hub genes by Yiqi Gubiao pills. The mRNA levels and protein expression levels of AKT1, ALB, TP53, IL6 and MAPK3 were detected using RT-qPCR and western blotting, respectively, to further validate the effects of Yiqi Gubiao pills on the predicted hub genes. The mRNA expression of AKT1, ALB, TP53, IL6 and MAPK3 was significantly suppressed by Yiqi Gubiao pills in a concentration-dependent manner (all $\mathrm{P}<0.05$ or $\mathrm{P}<0.01$; Fig. 5). Western blotting further revealed that the extract of the Yiqi Gubiao pills modulated protein expression of AKT1, ALB, TP53, IL6 and MAPK3. The protein expression levels of the TP53 and MAPK3 were significantly decreased in the Yiqi Gubiao pill groups compared with the control group, while protein expression levels of the AKT1, ALB and IL- 6 were not improved at Yiqi Gubiao Pills (12.5 $\mu \mathrm{g} / \mathrm{ml}$ ) (Fig. 6).

\section{Discussion}

Yiqi Gubiao pills are composed of 13 TCMs used to treat a variety of lung diseases. They are often used as an adjunct treatment in clinical treatment of $\operatorname{LC}(5,6)$. Preparation of TCM involves a combination of multiple ingredients of TCM. This in turn causes the action mechanism of TCMs to be complex because each component corresponds to several targets (19). Network pharmacology is performed to search for TCM ingredients, ingredient targets and disease targets in verified results of clinical trials $(8,20)$, which may systematically reveal the interaction between the ingredients and the diseases as well as the mechanisms of action of TCM in treating diseases (21).

In the present study, 102 active ingredients of Yiqi Gubiao pills were identified including 7 ingredients of BZ, 8 ingredients of BX, 4 ingredients of $\mathrm{CP}, 12$ ingredients of DS, 11 ingredients of FF, 7 ingredients of FL, 2 ingredients of FXM, 19 ingredients of HQ, 8 ingredients of KDH, 8 ingredients of PPY, 6 ingredients of YBM, 4 ingredients of YYR and 6 ingredients of ZS. Moreover, 2,658 ingredient targets were obtained after deleting the duplicate targets and 2,413 disease-related targets were identified. The disease related targets were further intersected to obtain 229 common targets. The 229 targets were regarded as potential targets for the Yiqi Gubiao pills in LC treatment. The PPI network of the hub genes further revealed that ATK1, TP53, ALB, IL6 and MAPK3 were the top five hub genes associated with LC treatment. These results were further verified using western blotting and RT-qPCR experiments.

Zhang et al (22) reported that PRMT5 activates AKT and induces the positive regulator PI3K and the negative regulator PTEN thereby inhibiting LC cell proliferation. In addition, Olivier et al (23) reported that TP53 is a key tumor suppressor gene. As such, its mutation may lead to the loss of function to inhibit tumor growth, thereby promoting cancer cell proliferation and cell invasion. Similarly, Zheng et al (24) suggested that upregulation of long non-coding RNA H19 induces the expression of microRNA (miR)-675-5p that targets the TP53 gene. This promotes tumor progression and development in NSCLC. The level of ALB is usually regarded as the key parameter that reflects an individual's nutritional status. The ratio of albumin-to-fibrinogen has been used as a biomarker to judge the clinical prognosis of patients with NSCLS (25). Studies have reported that the ERK1 signaling pathway plays a key role in promoting the proliferation of NSCLC mesenchymal stem cells $(26,27)$. As such, blocking the ERK signaling pathway improves the therapeutic response of patients with NSCLC to EGFR inhibitors (28). In the same line, the IL6 family of proteins is mainly involved in inflammation, immune response and development of tumors, thus leading to NSCLC progression (29). Yang et al have reported that miR-218 targets the IL6/STAT3 signaling pathway can prevent LC progression.

Targets of the Yiqi Gubiao pills were mainly enriched in the PI3K-Akt and AGE-RAGE signaling pathways. The PI3K-Akt signaling pathway is an intracellular signaling pathway mainly involved in the cell cycle process. It is related to cellular quiescence, proliferation, cancer and longevity (30). Wu et al (31) reported that the PAX6-zinc finger E-box-binding homeobox 2 axis promotes tumor metastasis via the PI3K/AKT signaling pathway. On the other hand, the AGE-RAGE signaling pathway is an important signal transduction pathway. It is mainly involved in major mechanisms of vascular oxidative stresses through activation of the MAPK and NF- $\kappa$ B pathways (32). Zeng et al postulated that RHOJ mediates the AGE-RAGE signaling pathway in NSCLC (33).

Overall, in the present study network pharmacology was used to decipher the active ingredients and potential targets of the Yiqi Gubiao pill, thus enabling further related studies. The results suggested that Yiqi Gubiao pills partially inhibit 

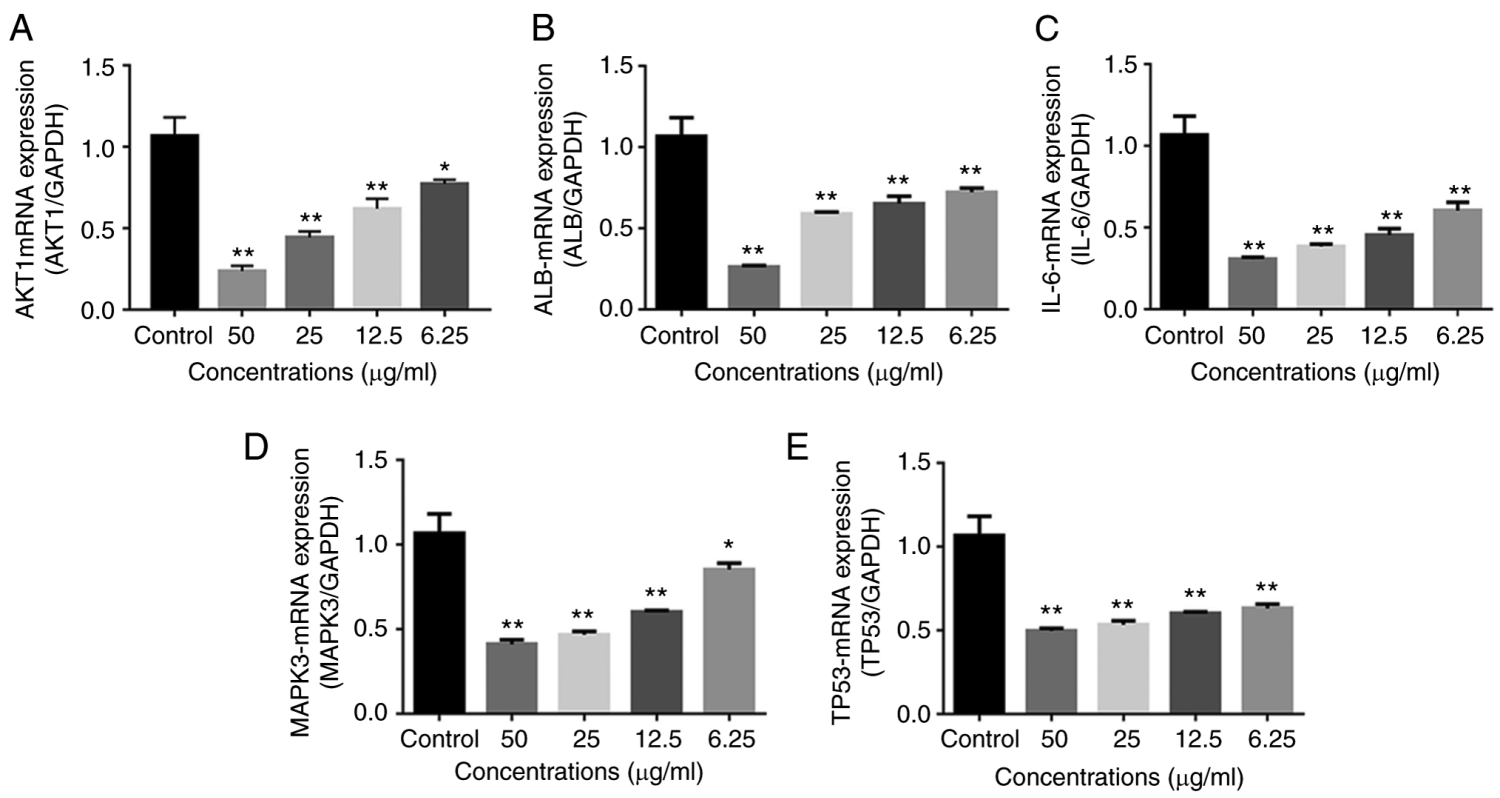

Figure 5. Yiqi Gubiao pill reduces the mRNA expression of hub genes, including (A) AKT1, (B) ALB, (C) IL-6, (D) MAPK3 and (E) and TP53. Data are expressed the mean \pm standard error $(n=3)$. ${ }^{*} \mathrm{P}<0.05$ and ${ }^{* *} \mathrm{P}<0.01$ vs. respective control.
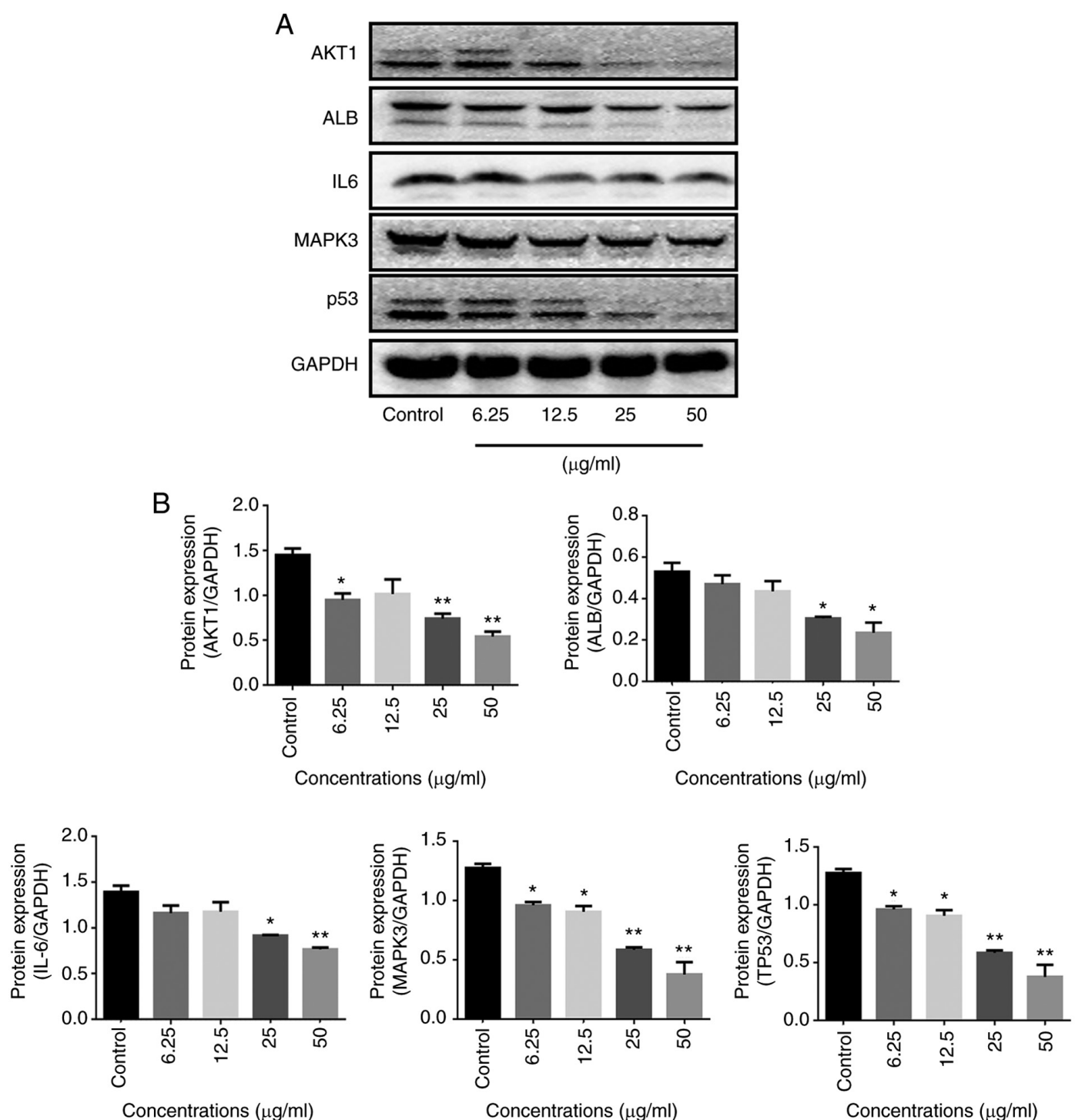

Figure 6. Expression levels of AKT, TP53, ALB, IL6 and MAPK3 were measured using (A) western blot analysis and (B) quantified. "P<0.05 and ${ }^{* *} \mathrm{P}<0.01$ 
progression of LC by regulating the expression of hub genes (AKT1, TP53, ALB, IL6 and MAPK3) through the PI3K/AKT and AGE-RAGE signaling pathways. The findings of the study provide a theoretical basis for the clinical application of Yiqi Gubiao pills in LC treatment.

\section{Acknowledgements}

Not applicable.

\section{Funding}

This study was supported by the Opening Project of the Key Laboratory of Xinjiang Uygur Autonomous Region (grant no. 2018D04004), and the Special Project for Young Medical Science and Technology Talents of the Health Commission of Xinjiang Uygur Autonomous Region (grant no. WJWY-201812).

\section{Availability of data and materials}

The datasets used and/or analyzed during the present study are available from the corresponding author upon reasonable request.

\section{Authors' contributions}

ZL made substantial contributions to the conception and performed the experiments. DX and JJ analyzed the data and revised the manuscript. FL gave final approval of the version to be published, made substantial contributions to design, and revised the manuscript critically for important intellectual content. ZL, DX, JJ and FL confirmed the authenticity of all the raw data. All authors have read and approved the final manuscript.

\section{Ethics approval and consent to participate}

Not applicable.

\section{Patient consent for publication}

Not applicable.

\section{Competing interests}

The authors declare that they have no competing interests.

\section{References}

1. Mao Y, Yang D, He J and Krasna MJ: Epidemiology of lung cancer. Surg Oncol Clin N Am 25: 439-345, 2016.

2. Rivera GA and Wakelee H: Lung cancer in never smokers. Adv Exp Med Biol 893: 43-57, 2016.

3. Glinski K, Socha J, Wasilewska-Tesluk E, Komosinska K and Kepka L: Accelerated hypofractionated radiotherapy with concurrent full dose chemotherapy for locally advanced non-small cell lung cancer: A phase I/II study. Radiother Oncol 148: 174-180, 2020

4. Kehoe S, Hook J, Nankivell M, Jayson GC, Kitchener H, Lopes T, Luesley D, Perren T, Bannoo S, Mascarenhas M, et al: Primary chemotherapy versus primary surgery for newly diagnosed advanced ovarian cancer (CHORUS): An open-label, randomised, controlled, non-inferiority trial. Lancet 386: 249-257, 2015.
5. Li FS, Zhang YL, LiZ, Xu D, Liao CY, Ma H, Gong L, Su J, Sun Q, Xu Q, et al: Randomized, double-blind, placebo-controlled superiority trial of the Yiqigubiao pill for the treatment of patients with chronic obstructive pulmonary disease at a stable stage. Exp Ther Med 12: 2477-2488, 2016.

6. Jiang L, Li F, Sun J, Huo H, Li X and Li H: Efficacy of Yiqigubiao pill on chronic obstructive pulmonary disease in rats with the disease induced by lipopolysaccharide and cigarette-smoke fumigation. J Tradit Chin Med 40: 983-991, 2020.

7. Boezio B, Audouze K, Ducrot P and Taboureau O: Network-based approaches in pharmacology. Mol Inform 36, 2017.

8. Kibble M, Saarinen N, Tang J, Wennerberg K, Mäkelä S and Aittokallio T: Network pharmacology applications to map the unexplored target space and therapeutic potential of natural products. Nat Prod Rep 32: 1249-1266, 2015.

9. Wu Y, Zhang F, Yang K, Fang S, Bu D, Li H, Sun L, Hu H, Gao K, Wang W, et al: SymMap: An integrative database of traditional Chinese medicine enhanced by symptom mapping. Nucleic Acids Res 47 (D1): D1110-D1117, 2019.

10. Ye H, Ye L, Kang H, Zhang D, Tao L, Tang K, Liu X, Zhu R, Liu Q, Chen YZ, et al: HIT: Linking herbal active ingredients to targets. Nucleic Acids Res 39 (Database Issue): D1055-D1059, 2011.

11. Keiser MJ, Roth BL, Armbruster BN, Ernsberger P, Irwin JJ and Shoichet BK: Relating protein pharmacology by ligand chemistry. Nat Biotechnol 25: 197-206, 2007.

12. Amberger JS, Bocchini CA, Schiettecatte F, Scott AF and Hamosh A: OMIM.org: Online mendelian inheritance in man $\left(\mathrm{OMIM}^{\circledR}\right)$, an online catalog of human genes and genetic disorders. Nucleic Acids Res 43 (Database Issue): D789-D798, 2015.

13. Zhou J and Rudd KE: EcoGene 3.0. Nucleic Acids Res 41 (Database Issue): D613-D624, 2013.

14. The UniProt Consortium: UniProt: The universal protein knowledgebase. Nucleic Acids Res 45 (D1): D158-D169, 2017.

15. Shannon P, Markiel A, Ozier O, Baliga NS, Wang JT, Ramage D, Amin N, Schwikowski B and Ideker T: Cytoscape: A software environment for integrated models of biomolecular interaction networks. Genome Res 13: 2498-2504, 2003.

16. The Gene Ontology Consortium: Expansion of the gene ontology knowledgebase and resources. Nucleic Acids Res 45 (D1): D331-D338, 2017.

17. Yu G, Wang LG, Han Y and He QY: clusterProfiler: An R package for comparing biological themes among gene clusters. OMICS 16: 284-287, 2012.

18. Livak KJ and Schmittgen TD: Analysis of relative gene expression data using real-time quantitative PCR and the 2(-Delta Delta C(T)) method. Methods 25: 402-408, 2001.

19. Kang Y,Zhu X, Xu Y, Tang Q, Huang Z, Zhao Z, Lu J, Song G, Xu H, Deng $C$ and Wang J: Energy stress-induced lncRNA HAND2-AS1 represses HIF1 $\alpha$-mediated energy metabolism and inhibits osteosarcoma progression. Am J Cancer Res 8: 526-537, 2018.

20. Li S and Zhang B: Traditional Chinese medicine network pharmacology: Theory, methodology and application. Chin J Nat Med 11: 110-120, 2013.

21. Zhang R, Zhu X, Bai H and Ning K: Network pharmacology databases for traditional Chinese medicine: Review and assessment. Front Pharmacol 10: 123, 2019.

22. Zhang S, Ma Y, Hu X, Zheng $\mathrm{Y}$ and Chen X: Targeting PRMT5/Akt signalling axis prevents human lung cancer cell growth. J Cell Mol Med 23: 1333-1342, 2019.

23. Olivier M, Hollstein M and Hainaut P: TP53 mutations in human cancers: Origins, consequences, and clinical use. Cold Spring Harb Perspect Biol 2: a001008, 2010.

24. Zheng ZH, Wu DM, Fan SH, Zhang ZF, Chen GQ and Lu J: Upregulation of miR-675-5p induced by lncRNA H19 was associated with tumor progression and development by targeting tumor suppressor p53 in non-small cell lung cancer. J Cell Biochem 120: 18724-18735, 2019.

25. Du X, Shao Y, Qin HF, Tai YH and Gao HJ: ALK-rearrangement in non-small-cell lung cancer (NSCLC). Thorac Cancer 9: 423-430, 2018.

26. Wang J, Zhou Y, Ma L, Cao S, Gao W, Xiong Q, Wang K and Yang L: CIAPIN1 targeted NHE1 and ERK1/2 to suppress NSCLC Cells' metastasis and predicted good prognosis in NSCLC patients receiving pulmonectomy. Oxid Med Cell Longev 2019: 1970818, 2019. 
27. Moncho-Amor V, Pintado-Berninches L, Ibañez de Cáceres I, Martín-Villar E, Quintanilla M, Chakravarty P, Cortes-Sempere M, Fernández-Varas B, Rodriguez-Antolín C, de Castro J, et al: Role of dusp6 phosphatase as a tumor suppressor in non-small cell lung cancer. Int J Mol Sci 20: 2036, 2019.

28. Sun C, Li C, Li X, Zhu Y, Su Z, Wang X, He Q, Zheng G and Feng B: Scutellarin induces apoptosis and autophagy in NSCLC cells through ERK1/2 and AKT signaling pathways in vitro and in vivo. J Cancer 9: 3247-3256, 2018.

29. Tang H, Bai Y, Pan G, Wang X, Wei Y, Yang Z and Zhao J: Interleukin- 6 and insulin-like growth factor-1 synergistically promote the progression of NSCLC. Autoimmunity 51: 399-407, 2018.

30. Xie Y, Shi X, Sheng K, Han G, Li W, Zhao Q, Jiang B, Feng J, $\mathrm{Li} \mathrm{J}$ and $\mathrm{Gu}$ Y: PI3K/Akt signaling transduction pathway, erythropoiesis and glycolysis in hypoxia (Review). Mol Med Rep 19 783-791, 2019.
31. Wu DM, Zhang T, Liu YB, Deng SH, Han R, Liu T, Li J and $\mathrm{Xu}$ Y: The PAX6-ZEB2 axis promotes metastasis and cisplatin resistance in non-small cell lung cancer through PI3K/AKT signaling. Cell Death Dis 10: 349, 2019.

32. Sparvero LJ, Asafu-Adjei D, Kang R, Tang D, Amin N, Im J, Rutledge R, Lin B, Amoscato AA, Zeh HJ and Lotze MT: RAGE (receptor for advanced glycation endproducts), RAGE ligands, and their role in cancer and inflammation. J Transl Med 7: 17, 2009.

33. Zeng T, Chen C, Yang P, Zuo W, Liu X and Zhang Y: A protective role for RHOJ in nonsmall cell lung cancer based on integrated bioinformatics analysis. J Comput Biol 27: 1092-1103, 2020.

(i) $($ ) This work is licensed under a Creative Commons Attribution-NonCommercial-NoDerivatives 4.0 International (CC BY-NC-ND 4.0) License. 